\title{
The Specificity and Sensitivity of PRRSV Aptamer using Quartz Crystal Microbalance (QCM)
}

\author{
Chakpatch Kuitio ${ }^{1}$, Peter Lieberzeit ${ }^{2}$, Kiattawee Choowongkumon ${ }^{3}$ \\ ${ }^{I}$ Genetic Engineering, Faculty of Graduate School, Kasetsart University, Bangkok, Thailand, \\ ${ }^{2}$ Department of Physical Chemistry, University of Vienna, Vienna Austria \\ ${ }^{3}$ Department of biochemistry, Faculty of science, Kasetsart University, Bangkok, Thailand*Corresponding \\ author.E-mail address: kiattawee.c@ku.th.
}

\begin{abstract}
The procine reproductive and respiratory syndrome (PRRS) cause by RNA virus, that is the important disases make economic lose in swine industry. The best for prevention from virus is screening swine befor come to the fram. Aptamer is a short ss-DNA that can bind with small molecule, cell, bacteria and virus, so the aptamer colud be develop to biosensor for screening the infection of procine reproductive and respiratory syndrome virus (PRRSV) in swine. In this study, want to test the specificity and sensitivity of PRRSV aptamer (7R) by quarzt crystal microbalance (QCM). The result from QCM show 7R aptamer could be bind with PRRSV around $10^{10}$ virus particles and 7R aptamer cloud not bind with Pseudorabies virus (PRV) and Classical swine fever vius (CSFV).
\end{abstract}

Key words: Aptamer, Quarzt Crystal Microbalance (QCM), Procine Reproductive and Respiratory Syndrome (PRRS)

\section{Introduction}

Porcine reproductive and respiratory syndrome is an important disease in swine industry cause by porcine reproductive and respiratory syndrome virus. The virus diagnosis by PCR, RT-PCR, ELISA and immunohistochemistry [1]. However the diagnosis of virus take a long time for result. So development of method for diagnosis is important. The aptamers are alternative choices for development of biosensor. The aptamers are single-strand deoxyribonucleic acid (ssDNA), that can be bound the target molecule with high specificity and affinity such as ions, drugs, toxins, proteins, cells, bacteria, and viruses. The Advantages of aptamers are can chemical synthesis, long-term storage, and easy to modification[2]. 7R aptamer are specific for PRRSV, selected by capillary electrophoresis and colorimetric method from previous experiment. In this study used the quartz crystal microbalance (QCM), interesting technique choice for detecting interaction of nucleotides and target [3] so QCM can used for observes specificity and sensitivity of $7 \mathrm{R}$ aptamer.

\section{Material and Method}

\section{DNA aptamer}

7R aptamer contained 40-mer modified at 5' end by thiol group (-SH). The sequence following sequence 5'-(ThiC6) GCTGTACCGT CTGCTAGGACACCATAACTTCTAGCAAACG C. The aptamer has been synthesized by SIGMA-ALDRICH.

\section{Quarzt Crystal Microbalance (QCM)}

Gold-electrode coated on $10 \mathrm{MHz}$ quartz and dropped 7R aptamer diluted with cysteine (1:1000) on working site of gold-electrode. And reference site dropped non-specific have 40mer diluted with cysteine same concentration of 7R aptamer. Baseline signal used $1 \mathrm{mM}$ TNE buffer $\mathrm{pH} 7.4$ until reaching the equilibrium state. The QCM system test with PRRSV around $10^{10}-10^{11}$ particles and test specificity with PRV in same concentration of viral particles.

\section{Result and Dicussion}

\section{Sequence analysis}

7R specific aptamer of PRRSV from previous studied by callpillary electrophoresis combined with coloremetric metod. That present good property for develop to bio-senser becase from 
sequencing, 7R aptamer contian 40-mer of nucleotide, shown one loop in secondary structure (Fig.2). The loop of aptamer are important role for interaction with a virus particel that facilitates formation of electrostatic interactions or hydrogen bonds between the aptamer and its target, as well as stacking interactions between aromatic compounds and the nucleobases[6].

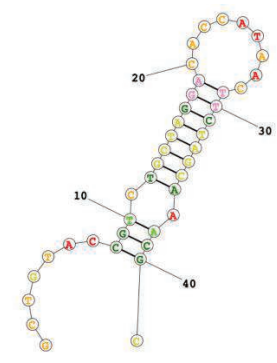

Figure2. The secondary structure of $7 R$ aptamer analysis by https://rna.urmc. rochester.edu

The sensitivity test of $7 \mathrm{R}$ aptamer using QCM

Gold-electrode coated with 7R aptamer solution in cysteine on working site and nonspecific DNA on reference site. PRRSV diluted with $1 \mathrm{mM}$ TNE bufer. The result from QCM shown in figure3, when add PRRSV $1.87^{*} 10^{10}$ particles frequency shift form baseline at

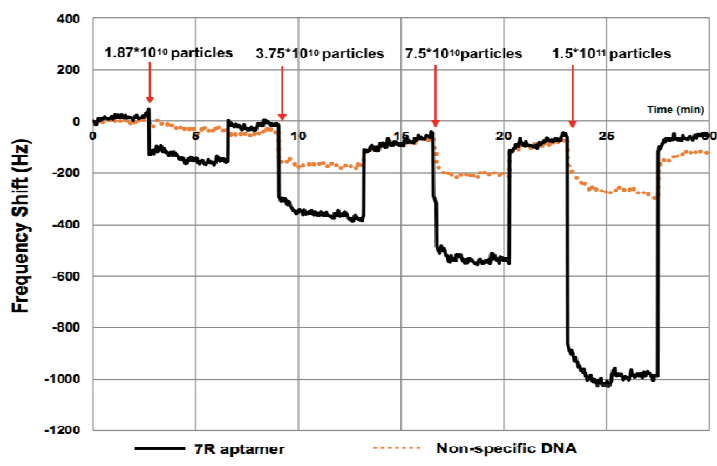

Figure3. The frequency shift form QCM tested with PRRSV $1.87 * 10^{10}$ particles $-1.5 * 10^{11}$ particles.

working site $150 \mathrm{~Hz}$. After flushing the system with mixed $10 \%$ aqueous solution of acetic acid, followed by deionized water, the virus particles are removed out and the frequency increases to baseline revealing fully reversibility. The frequency shift increase when add higher concentration of PRRSV $3.75^{*} 10^{10}, 7.5^{*} 10^{10}$ and $1.5^{*} 10^{11}$ particles frequency shift form baseline $402 \mathrm{~Hz}, 613 \mathrm{~Hz}$ and $1,046 \mathrm{~Hz}$ respectively correspond to mass loading. But at reference site frequency shift less than when compare whit working site. So the result could be indicate that, 7R aptamer can bind with PRRSV and can remove virus particle by acetic acid solution because structure of DNA aptamer will be change conformation when $\mathrm{pH}$ of solution change.

\section{The specificity test of $7 R$ aptamer using QCM}

Actually, In this study used CSFE and PRV for the specificity of $7 R$ aptamer, the result show when add CSFV and PRV around $1.5^{*} 10^{11}$ particles the frequency increase $95 \mathrm{~Hz}$ and then add PRV $1.5^{*} 10^{11}$ particles presented frequency shift $112 \mathrm{~Hz}$ from baseline. However when add PRRSV $1.5^{*} 10^{11}$ particles target of 7R aptamer, the frequency increase $1,322 \mathrm{~Hz}$ that have significant difference when compared with CSFV and PRV. So result indicated 7R aptamer specific to PRRSV and could not bind with CSFV and PRV.

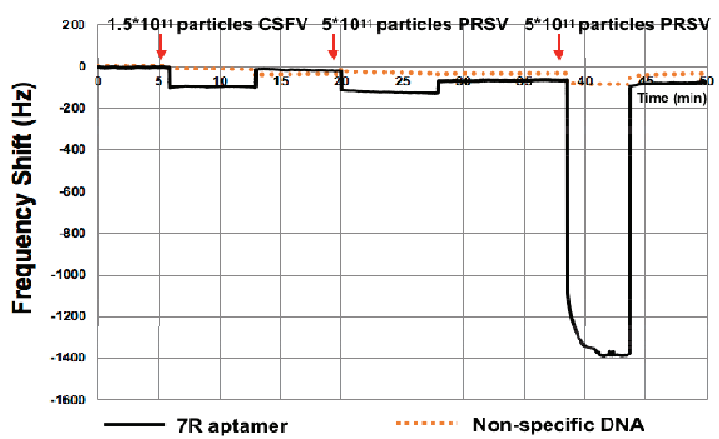

Figure4. The frequency shift form QCM tested with CSFV, PRV, PRRSV at $1.87 * 10^{10}$ particles $-1.5^{*} 10^{11}$ particles.

\section{Conclusions}

All results indicate successful for used QCM technique test specificity and sensitivity of aptamer because QCM can detected mass of target by frequency shift. The result shown QCM is appropriate for screening aptamer and test the property for development bio-sensor best on aptamer.

Acknowledgments: This project was supported by the Research and Researchers for Industries-RRIRoyal Thai Government, Thailand, the ASEAN-European Academic University Network (ASEA-UNINET).

\section{References}

[1] Lunney JK, Fang Y, Porcine Reproductive and Respiratory Syndrome Virus (PRRSV): Pathogenesis and Interaction with the Immune System, Annu Rev Anim Biosci 4, 129-54 (2016); doi: 10.1146/annurevanimal-022114-111025.

[2] A.V. Lakhin, V.Z. Tarantul, and L.V. Gening, Aptamers: Problems, Solutions and Prospects, Acta Naturae 5(4), 34-43 (2013)

[3] Kespunyavee Bunroddith, Peter Lieberzeit, QCMbased rapid detection of PCR amplification products of Ehrlichia canis, Analytica Chimica Acta 11, 106111(2018); doi: 10.1016/j.aca.2017.10.037 